\title{
Phase-contrast magnetic resonance imaging reveals net retrograde aqueductal flow in idiopathic normal pressure hydrocephalus
}

\author{
Geir Ringstad, MD, ${ }^{1}$ Kyrre Eeg Emblem, PhD, ${ }^{2}$ and Per Kristian Eide, MD, PhD ${ }^{3,4}$ \\ 'Department of Radiology and Nuclear Medicine, ${ }^{2}$ The Intervention Centre, and ${ }^{3}$ Department of Neurosurgery, Oslo University \\ Hospital-Rikshospitalet, and ${ }^{4}$ Faculty of Medicine, University of Oslo, Oslo, Norway
}

\begin{abstract}
OBJECTIVE The objective of this study was to assess the net aqueductal stroke volume (ASV) and CSF aqueductal flow rate derived from phase-contrast MRI (PC-MRI) in patients with probable idiopathic normal pressure hydrocephalus (iNPH) before and after ventriculoperitoneal shunt surgery, and to compare observations with intracranial pressure (ICP) scores.
\end{abstract}

METHODS PC-MRI at the level of the sylvian aqueduct was undertaken in patients undergoing assessment for probable iNPH. Aqueductal flow in the craniocaudal direction was defined as positive, or antegrade flow, and net ASV was calculated by subtracting retrograde from antegrade aqueductal flow. Aqueductal flow rate per minute was calculated by multiplying net ASV by heart rate. During the same hospital admission, clinical examination was performed using $\mathrm{NPH}$ score and overnight continuous ICP monitoring. Twelve patients were followed prospectively 12 months after shunt placement with clinical assessment and a second PC-MRI. The study also included 2 healthy controls.

RESULTS Among 21 patients examined for iNPH, $17(81 \%)$ received a shunt (shunt group), and 4 were treated conservatively (conservative group). Among the patients with shunts, a clinical improvement was observed in 16 (94\%) of the 17. Net ASV was negative in $16(76 \%)$ of 21 patients before shunt placement and in $5(42 \%)$ of 12 patients after shunt placement, and increased from a median of $-5 \mu \mathrm{l}$ (range -175 to $27 \mu \mathrm{l}$ ) to a median of $1 \mu \mathrm{l}$ (range -61 to $30 \mu \mathrm{l} ; p=$ 0.04). Among the 12 patients with PC-MRI after shunt placement, 11 were shunt responders, and in 9 of these 11 either a reduced magnitude of retrograde aqueductal flow, or a complete reversal from retrograde to antegrade flow, occurred. Net ASV was significantly lower in the shunt group than in the conservative group $(p=0.01)$. The aqueductal flow rate increased from $-0.56 \mathrm{ml} / \mathrm{min}$ (range -12.78 to $0.58 \mathrm{ml} / \mathrm{min}$ ) to $0.06 \mathrm{ml} / \mathrm{min}$ (range -4.51 to $1.93 \mathrm{ml} / \mathrm{min} ; p=0.04$ ) after shunt placement.

CONCLUSIONS In this cohort of patients with iNPH, retrograde net aqueductal flow was observed in $16(76 \%)$ of 21 patients. It was reversed toward the antegrade direction after shunt placement either by magnitude or completely in 9 $(75 \%)$ of 12 patients examined using PC-MRI both before and after shunt placement $(p=0.04) ; 11$ of the 12 were shunt responders. The study results question previously established concepts with respect to both CSF circulation pathways and CSF formation rate.

http://thejns.org/doi/abs/10.3171/2015.6.JNS15496

KEY WORDS idiopathic normal pressure hydrocephalus; aqueductal flow; phase-contrast MRI; shunt surgery

$\mathbf{P}$ HASE-CONTRAST MRI (PC-MRI) can be used to quantify CSF flow at the level of the aqueduct by synchronizing the acquisition of the images with the cardiac cycle..$^{32}$ Net retrograde aqueductal flow has been demonstrated with PC-MRI in a few studies with limited numbers $(\mathrm{n}=2-11)$ of both patients with hydrocephalus ${ }^{18,27,33}$ and healthy infants. ${ }^{7}$ These observations are contradictory to the established concept of CSF circulation of antegrade, bulk flow from the ventricles toward the pacchionian granulations at the surface of the brain. The magnitude of retrograde aqueductal flow rates in these studies also implies CSF formation rates that are higher than what has been established previously from studies utilizing invasive methods, and also with PC-MRI in healthy adults. One important objection to these controversial observations is that measurements of minute quantities of net aqueductal flow

ABBREVIATIONS AQP4 = aquaporin 4; ASV = aqueductal stroke volume; ICP = intracranial pressure; iNPH = idiopathic NPH; MWA = mean ICP wave amplitudes; NPH = normal pressure hydrocephalus; PC-MRI = phase-contrast $\mathrm{MRI}$; ROI = region of interest.

SUBMITTED March 3, 2015. ACCEPTED June 4, 2015.

INCLUDE WHEN CITING Published online December 4, 2015; DOI: 10.3171/2015.6.JNS15496. 
with PC-MRI may be technically challenging ${ }^{25}$ and more sensitive to error than measurements of mean aqueductal flow (aqueductal stroke volume [ASV]), which have been proposed as a tool to predict shunt response in normal pressure hydrocephalus (NPH). ${ }^{11}$

However, in recent years, an increasing awareness of our incomplete understanding of CSF circulation has arisen, ${ }^{12}$ and data from animal studies have demonstrated that water exchange between the paravascular and interstitial space may play a crucial role in both hydrocephalus and clearance of waste products from brain metabolism. ${ }^{10,26} \mathrm{In}$ this context, the previous observations of retrograde aqueductal CSF flow should be further explored.

Our study was performed in a cohort of patients with probable idiopathic NPH (iNPH) before and after ventriculoperitoneal shunt surgery. The objective was to determine the net aqueductal flow by PC-MRI-derived net ASV and estimate the CSF aqueductal flow rate in patients with probable iNPH before and after ventriculoperitoneal shunt surgery, and compare observations with intracranial pressure (ICP) scores.

\section{Methods}

\section{Patient Inclusion and Study Design}

The Regional Ethics Committee, REK South-East, and Institutional Review Board approved this prospective study. Written and oral informed consent was obtained from 28 patients undergoing assessment for probable iNPH within the neurosurgical department. The study included 21 consecutive patients with successfully obtained PC-MRI. The 7 unsuccessful examinations were due to termination of the examination before completion by the patient or technician, or motion artifacts disturbing the PC-MR image quality. In addition to PC-MRI, the patient workup also included a clinical examination with determination of severity of iNPH symptomatology, and overnight ICP monitoring, including determination of the mean ICP wave amplitudes (MWAs). All patients with iNPH who received shunts were offered a second PC-MRI 12 months after surgery.

Two healthy controls underwent PC-MRI for validation of the aqueductal flow measurements. A subset of data from the patient cohort with focus on the utility of ASV (mean ASV) in diagnosing reduced intracranial compliance has been presented previously. ${ }^{34}$

\section{Clinical Management}

Management of patients with iNPH followed the clinical routine at the department of neurosurgery. The severity of symptoms was graded using an NPH grading scale, ${ }^{22}$ which assesses the combined severity of gait disturbance, urinary incontinence, and dementia. Each component is graded from 1 to 5 , which gives a total score from 3 (worst) to 15 (best). According to this department's routine, the indication for shunt surgery is based on a combination of clinical assessment, radiological assessment, and the results of continuous ICP monitoring, as previously described. ${ }^{22}$ The shunt response was defined as an improvement of at least 2 points in the NPH score, and was assessed regularly at 3,6, and 12 months after shunting.
The neurosurgeon who performed the clinical assessment (P.K.E.) was blinded to all PC-MRI data.

\section{PC-MRI Technique}

All PC-MRI data were acquired on a 3-T MRI scanner (Philips Achieva, Philips Medical Systems) with a 16-channel head coil, and an acquisition plane perpendicular to the aqueduct, with the following parameters: TR = $24 \mathrm{msec}, \mathrm{TE}=16 \mathrm{msec}$, voxel size $0.60 \times 0.80 \times 4.00 \mathrm{~mm}^{3}$, matrix $232 \times 175$ pixels, FOV $14 \mathrm{~cm}$, velocity encoding 10 $\mathrm{cm} / \mathrm{sec}$, and $30-40$ phases with retrospective peripheral cardiac gating.

As part of the postprocessing routine, a region of interest (ROI) was manually defined along the outer border of the aqueduct (Fig. 1) by an experienced neuroradiologist (G.R.), using Philips Q-flow software (Philips Medical Systems). The neuroradiologist was blinded to information about clinical NPH score and whether the patient had responded to shunting or not.

Any flow aliasing was corrected by using a simple sinusoid curve alignment with second-order Fourier transformation. A constraint to the PC-MRI method and measurements of aqueductal flow is eddy currents, which may introduce a significant velocity phase offset and cause baseline shift artifacts in the MRI data. ${ }^{24,28}$ In our hospital, this had been corrected for by the vendor of the MRI machine. To minimize noise from nonmoving elements, care was taken to not include periaqueductal tissue in the ROI.

CSF flow in the craniocaudal direction was defined as positive, or antegrade, flow. Net ASV was calculated by subtracting the PC-MRI-derived volumetric estimate of retrograde aqueductal flow from antegrade flow through 1 cardiac cycle.

CSF net aqueductal flow rate per minute was calculated by multiplying net ASV by heart rate.

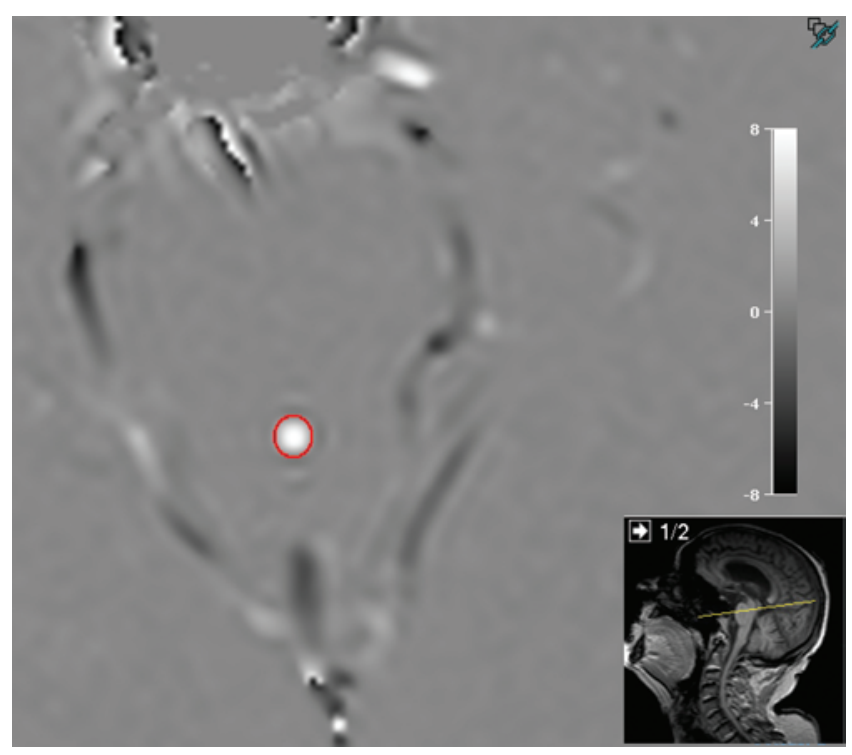

FIG. 1. PC-MRI was performed with slice orientation perpendicular to the aqueduct. The manually drawn ROI (red circle) defines the aqueduct from which the PC-MRI flow measurements are made. Figure is available in color online only. 


\section{ICP Monitoring}

Continuous overnight monitoring of ICP, including MWA, was performed in all patients. As previously described in detail, ${ }^{22}$ an ICP sensor was placed in the brain parenchyma through a small bur hole in the skull with the patient under local anesthesia. Overnight monitoring was conducted in the patient ward using a computerized system (Sensometrics AS, dPCom) for automatic identification of individual cardiac-induced single ICP waves. MWA is defined as the pressure difference between the systolic maximum and diastolic minimum pressures, ${ }^{22}$ and is determined as the average of all single ICP waves during consecutive 6 -second time intervals. The MWA values were determined for the 6-second time windows from 11 PM to 7 AM (i.e., 4800 6-second time windows). Both the average of MWA was determined, as well as the percentage of MWA, as $\geq 5 \mathrm{~mm} \mathrm{Hg}$ or $\geq 6 \mathrm{~mm} \mathrm{Hg}$ during the recording period.

Threshold levels of MWA representing an indication for shunt placement are MWA on average $\geq 4 \mathrm{~mm} \mathrm{Hg}$ (high MWA) and/or percentage of MWA $\geq 5 \mathrm{~mm} \mathrm{Hg}$ in $\geq$ $10 \%$ of recording time (labeled "high MWA" subgroup). ${ }^{22}$

\section{Statistical Analysis}

Values of net ASV and estimated CSF aqueductal flow rate for 12 patients before and after shunting were compared using a pairwise Wilcoxon signed-rank test. Different groups were compared using the Mann-Whitney U-test. The significance level was set to 0.05 . Statistical analysis was performed using SPSS statistical software (version 20, IBM Corp.).

\section{Results \\ Patients}

From the cohort of 21 patients with clinical signs of iNPH, 17 patients were selected for shunting, 16 of whom had high MWA. The 16 patients with high MWA (94\%) responded clinically by an increase in NPH score of at least 2 points, while 1 patient with low MWA (6\%) did not respond. Other patient data are presented in Table 1.

\section{Net ASV and Aqueductal Flow Rate Before and After Shunting}

Table 2 presents the net ASV values as well as the estimated daily CSF formation rates for all patients with iNPH and the 2 healthy individuals.
Before shunting, net ASV was a median $-5 \mu l$ (range -175 to $27 \mu \mathrm{l})$ and negative in $16(76 \%)$ of 21 patients. Of 17 patients who underwent shunt placement, 12 returned for a second PC-MRI session 12 months after shunting, where net ASV was increased to a median of $2 \mu \mathrm{l}$ (range -61 to $30 \mu \mathrm{l} ; \mathrm{p}=0.04)$ and was now negative in $5(42 \%)$ of 12 patients. Aqueductal flow rate increased from -0.56 $\mathrm{ml} / \mathrm{min}$ (range -12.78 to $0.58 \mathrm{ml} / \mathrm{min}$ ) before shunt placement to $0.06 \mathrm{ml} / \mathrm{min}$ (range -4.51 to $1.93 \mathrm{ml} / \mathrm{min}$ ) after shunt placement $(\mathrm{p}=0.04)$. Net ASV and aqueductal flow rate before and after shunting are presented in Fig. 2.

On the subgroup level, net ASV was lower in patients who received shunts (shunt group; $-11 \mu \mathrm{l}$ [range -175 to $11 \mu l], \mathrm{n}=17$ ) than in patients who did not receive shunts (conservative group; $9 \mu \mathrm{l}$ [range -4 to $27 \mu \mathrm{l}$ ], $\mathrm{n}=4$; $\mathrm{p}=$ $0.01)$. Aqueductal flow rate was also lower in the shunt group $(-0.77 \mathrm{ml} / \mathrm{min}$ [range -12.78 to $0.58 \mathrm{ml} / \mathrm{min}$ ]) than in the conservative group $(0.75 \mathrm{ml} / \mathrm{min}$ [range -0.24 to $2.04 \mathrm{ml} / \mathrm{min}$ ]; $\mathrm{p}=0.009$ ).

\section{Net Direction of Aqueductal Flow and MWA Subgroups}

Among the 21 patients with iNPH undergoing preoperative assessment of MWA, increased MWA values as defined in the Methods section (high MWA) were found in 17 patients and low MWA values in 4 patients. Prior to shunt placement, negative values of net ASV were found in $14(82 \%)$ of 17 patients with high MWA, and in $1(25 \%)$ of 4 with low MWA (Fig. 3 left). Net ASV at PC-MRI 12 months after surgery increased in $9(75 \%)$ of 12 patients in the high MWA cohort. In 1 of the 3 patients with a decline in net ASV after shunt placement, aqueductal flow had changed from being net antegrade to net retrograde (Fig. 2 upper), while the patient had responded clinically to shunt placement. However, this patient was in a severe preoperative clinical state (NPH score of 4 on a scale from 3 to 15), and following shunting, the NPH score was 8, still indicating a poor clinical condition. The estimated aqueductal flow rates in the MWA subgroups (high MWA/low MWA) are presented in Fig. 3 right.

\section{Discussion}

The main observation of this study was retrograde net aqueductal flow in the majority of patients with iNPH before shunt placement (Fig. 4) and a change toward antegrade flow after shunting.

TABLE 1. Patient characteristics

\begin{tabular}{|c|c|c|c|c|}
\hline \multirow[b]{2}{*}{ Variable } & \multicolumn{3}{|c|}{ iNPH } & \multirow[b]{2}{*}{ Healthy Controls } \\
\hline & Total & Shunt Group & Conservative Group & \\
\hline No. of patients & 21 & 17 & 4 & 2 \\
\hline Median age in yrs (range) & $74(56-84)$ & $74(56-84)$ & $74(60-79)$ & $50(45-54)$ \\
\hline F/M & $10 / 11$ & $8 / 9$ & $2 / 2$ & $2 / 0$ \\
\hline \multicolumn{5}{|l|}{ Preop clinical state (range) } \\
\hline Median NPH score & $10(4-13)$ & $10(4-13)$ & $11(9-13)$ & - \\
\hline Median duration of symptoms (yrs) & $2(0.5-10)$ & $2(1.0-10.0)$ & $0.8(0.5-10.0)$ & - \\
\hline \multicolumn{5}{|l|}{ Postop clinical state (range) } \\
\hline Median NPH score & $12(8-15)$ & $12(8-15)$ & - & - \\
\hline
\end{tabular}


TABLE 2. PC-MRI-derived measures and ICP scores*

\begin{tabular}{|c|c|c|c|c|}
\hline \multirow[b]{2}{*}{ Assessments } & \multicolumn{3}{|c|}{ iNPH } & \multirow[b]{2}{*}{ Healthy Controls $(n=2$} \\
\hline & Total $(n=21)$ & Shunt Group $(n=17)$ & Conservative Group $(n=4)$ & \\
\hline \multicolumn{5}{|l|}{ PC-MRI measures } \\
\hline \multicolumn{5}{|l|}{ Net ASV $(\mu \mathrm{l})$} \\
\hline Before shunt & $-5(-175$ to 27$)$ & $-11(-175$ to 11$) \S$ & $9(-4$ to 27$)$ & $3(2-3)$ \\
\hline After shunt $(n=12)$ & $2(-61$ to 30$)$ & $2(-61$ to 30$)$ & - & - \\
\hline \multicolumn{5}{|c|}{$\begin{array}{l}\text { Estimated CSF aqueductal } \\
\text { flow rate }(\mathrm{ml} / \mathrm{min}) \dagger\end{array}$} \\
\hline Before shunt & $-0.56(-12.78$ to 0.58$)$ & $-0.77(-12.78$ to 0.58$) \S$ & $0.75(-0.24$ to 2.04$)$ & $0.15(0.10-0.19)$ \\
\hline After shunt & $0.06(-4.51$ to 1.93$)$ & $0.06(-4.51$ to 1.93$)$ & - & - \\
\hline \multicolumn{5}{|l|}{ ICP scores $\ddagger$} \\
\hline \multicolumn{5}{|l|}{ Mean ICP $(\mathrm{mm} \mathrm{Hg})$} \\
\hline Average & $6.1(-1.8$ to 11.9$)$ & $6.1(-1.8$ to 11.9$)$ & $5.8(3.0-8.8)$ & - \\
\hline Percentage $>15$ & $0(0-2)$ & $0(0-2)$ & $0(0-0)$ & - \\
\hline \multicolumn{5}{|l|}{$\mathrm{MWA}(\mathrm{mm} \mathrm{Hg})$} \\
\hline Average & $4.5(3.1-7.9)$ & $4.7(3.5-7.9)$ ๆ & $3.4(3.1-5.2)$ & - \\
\hline Percentage $>5$ & $26(1-100)$ & $27(-100) \pi$ & $3(1-58)$ & - \\
\hline
\end{tabular}

* Data are presented as median values with ranges.

$\dagger$ Antegrade/craniocaudal flow is defined with positive values.

‡ ICP scores recorded from 11 PM to $7 \mathrm{AM}$.

$\S$ Significant difference between shunt and conservative groups before shunting $(p<0.02$, Mann-Whitney U-test).

II Significant difference between shunt and conservative groups before shunting ( $p<0.05$, Mann-Whitney U-test).

\section{Retrograde Aqueductal CSF Flow}

In healthy adults, antegrade aqueductal flow has been demonstrated in several studies using PC-MRI,,$^{25,27,33}$ and this could also be reproduced in our 2 healthy controls. A main observation of our study is net retrograde aqueductal flow in 16 of 21 patients with probable iNPH and a significant change in magnitude of net aqueductal flow toward the antegrade direction after shunting. Reversal of net retrograde aqueductal flow after shunting has previously been reported in smaller patient cohorts. ${ }^{4,27,33}$ The observed retrograde-directed flow supports an alternative circulation pathway for CSF in iNPH, as suggested by Penn et al., ${ }^{33}$ in which CSF is resorbed by the brain parenchyma through the ventricular ependyma and replenished at the outer surface of the brain and medulla. Retrograde aqueductal flow indicates a positive pressure gradient from the ventricles over the ependyma. Such a gradient can be sufficient to enlarge the ventricles ${ }^{29,30}$ and has been demonstrated previously by invasively measured pressure amplitudes in patients with hydrocephalus, which in 1 study was a median $0.4 \mathrm{~mm} \mathrm{Hg}$ higher in the ventricles than in the parenchyma. ${ }^{19}$ The same study also reported reversal of the pulse pressure amplitude difference after shunt placement, which is consistent with our findings of reversal from retrograde to antegrade aqueductal flow in patients with shunts. Reversal of aqueductal flow might also shed light on the mechanism behind ventricular enlargement and the shunt effect, as the positive pressure gradient from ventricle to parenchyma can be hypothesized to be reversed after shunting, and thereby facilitate antegrade flow and reduced ventricular size. A later study on 10 patients from the same research group ${ }^{20}$ however, was not able to demonstrate any pulse pressure gradients between the ventricles and the subdural compartment.

In obstructive hydrocephalus, an alternative pathway for absorption of CSF through the ventricular ependyma was proven decades ago ${ }^{9,36}$ and has later been supported by findings of elevated periventricular brain water content. ${ }^{10}$ Our observations of net retrograde aqueductal flow in iNPH suggest that this route for CSF resorption may take place in communicating hydrocephalus as well. Subsequently, CSF resorption through the ventricular ependyma requires that subarachnoid CSF must be replenished from the surface of the CNS, implying a net production of CSF outside the ventricles. This is supported by previous findings of CSF drainage by periarterial flow out of the brain parenchyma. ${ }^{15,38}$ In contrast, Iliff et al., ${ }^{26}$ using in vivo 2-photon imaging of small fluorescent tracers, recently demonstrated that CSF enters the parenchyma along paravascular spaces that surround penetrating arteries at the surface of the brain, and the clearance of brain water through the interstitial space toward paravenous routes ("the glymphatic system"). ${ }^{26}$ This model supports resorption, and not production, of CSF at the brain surface. However, it was not recorded under which pressure the tracer was injected, rendering the possibility that the tracer was forced in the opposite direction of physiological flow. In our study, we found that net aqueductal flow could be either antegrade or retrograde, which implies that net direction of CSF flow through the brain in both ways is possible, and could be changed by shunt placement. Accordingly, CSF circulation is fundamentally not restricted to one direction or the other, but may rather reflect flow along the pathway of least resistance. In disease with limited capacity for CSF resorption from the surface of the 


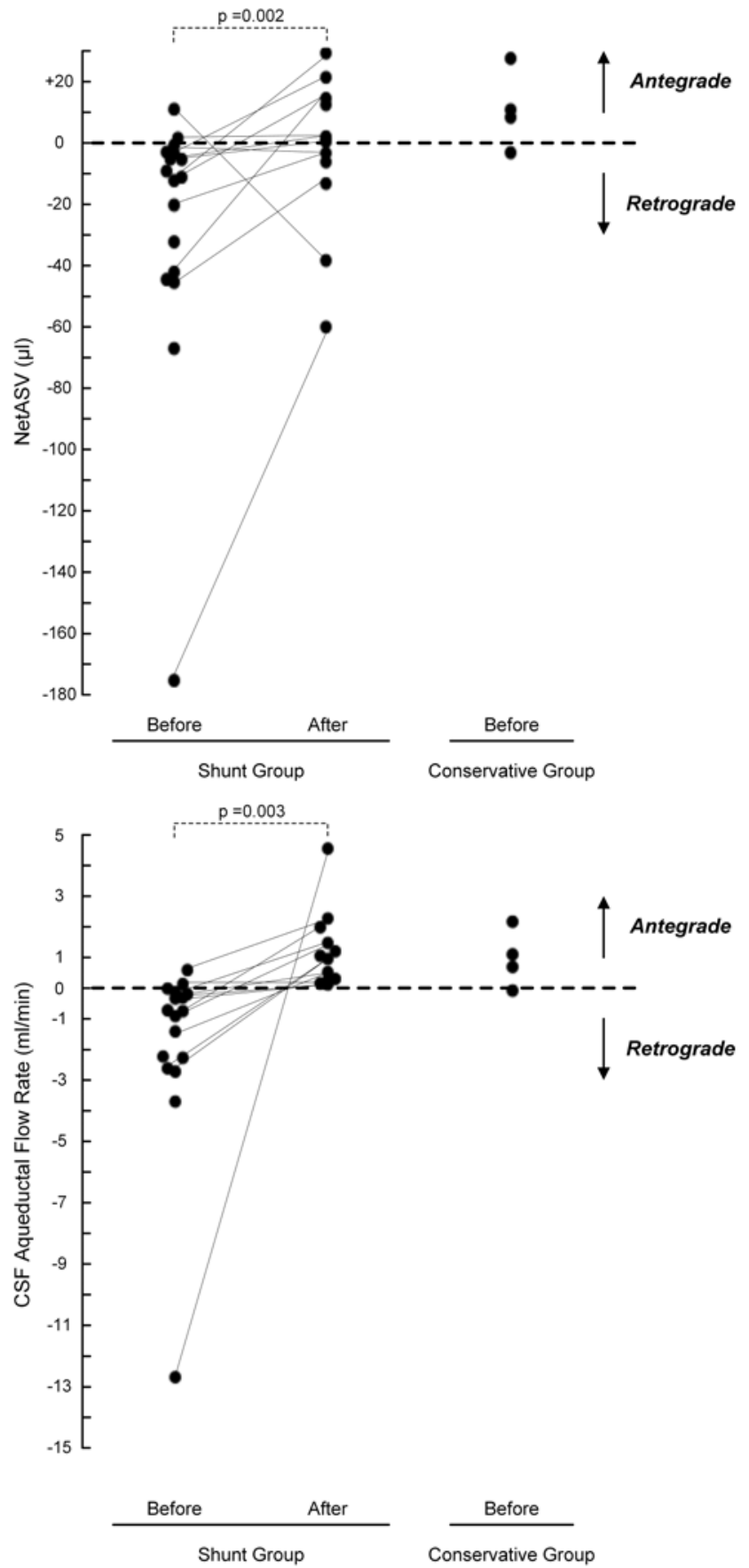

FIG. 2. Graphic presentation of the PC-MRI-derived indices net ASV ( $\mu$ l; upper) and estimated CSF net aqueductal flow rate ( $\mathrm{ml} / \mathrm{min}$; lower) for iNPH patients with shunts before $(n=17)$ and after $(n=12)$ receiving the shunts, and for conservatively managed patients with iNPH $(n=4)$. The $p$ values for differences between patients before and after shunt placement were determined by Wilcoxon signed-rank tests.

CNS, CSF flow might therefore be redirected into the ventricles and through the ventricular ependyma.

\section{CSF Formation Rate}

Since the experiments carried out by Dandy on $1 \mathrm{dog}$ in $1919,{ }^{17}$ for a long time the choroid plexus has been undisputed by most researchers as the major site of CSF production. More than 50 years ago, Rubin et al. used the ventriculocisternal perfusion technique on patients with tumors and found an average CSF formation rate of 0.37 $\mathrm{ml} / \mathrm{min}$ and a maximum absorption capacity of $1.3 \mathrm{ml} /$ min. ${ }^{35}$ Other studies have demonstrated values in the same range, ${ }^{16,31}$ and a more recent review article reported an average estimated CSF formation rate of $0.36 \mathrm{ml} / \mathrm{min} .^{13}$

A PC-MRI study of healthy patients suggested an average rate of $0.31 \mathrm{ml} / \mathrm{min} .{ }^{25}$ Aqueductal CSF flow rates in our study range from -12.78 to $1.93 \mathrm{ml} / \mathrm{min}$ and imply CSF formation rates that are profoundly different from those in previous reports.

While invasive indicator techniques for estimating CSF formation rates all use proteins and polar substances designed not to be absorbed into capillaries, ${ }^{6}$ a study performed already in $1952^{8}$ demonstrated that water exchanges freely and constantly between the blood, brain, and CSF. This was recently addressed in a review article by Brinker et al. ${ }^{12}$ The original data have recently been used to recalculate CSF flow by applying MRI-based volume assessments of the CSF spaces, and flow rates of more than $22 \mathrm{ml} / \mathrm{min}$ have been estimated. ${ }^{5}$ These values are also larger than the aqueductal flow rates in our cohort of patients with iNPH who did not receive a shunt.

In recent years, the astroglial aquaporin-4 (AQP4) water channel, which is polarized to perivascular astrocytic end feet, has been described in humans. ${ }^{1}$ This water channel is expressed at all borders between brain parenchyma and major fluid compartments, ${ }^{2}$ and acceleration of hydrocephalus has been shown in AQP4-null mice. ${ }^{10}$ Iliff et al. ${ }^{26}$ demonstrated that a substantial portion of subarachnoid CSF cycles through the interstitial space in the mouse brain, and also found an enlarged extracellular space in mice lacking the AQP4 gene. These observations, indicating that water flux takes place through the vast surface of the brain microvasculature, renders a potential for much larger CSF formation rates than what was previously believed to be limited by the surface of the arachnoid granulations.

\section{Net ASV and ICP Scores}

We compared net ASV with invasive ICP scores. The MWAs have proven to be a robust parameter of predicting shunt response in iNPH. ${ }^{22}$ The ICP wave amplitudes can be considered a surrogate marker of intracranial compliance (i.e., pressure-volume reserve capacity). ${ }^{21}$ Thus, increased MWA values would indicate impaired compliance, or reduced intracranial pressure-volume reserve capacity. The present observations of negative net aqueductal flow in 14 (82\%) of 17 patients with high MWA values suggest that impaired intracranial compliance is associated with net CSF flow into the ventricles.

\section{Methodological Limitations Regarding Measurements of Net ASV}

Aqueductal flow measurements have several intrinsic difficulties. The net ASV represents a very small quantity of flow derived from the subtraction of 2 considerably larger values of bidirectional, oscillating flow, and any imprecision in measurements of flow in the antegrade 

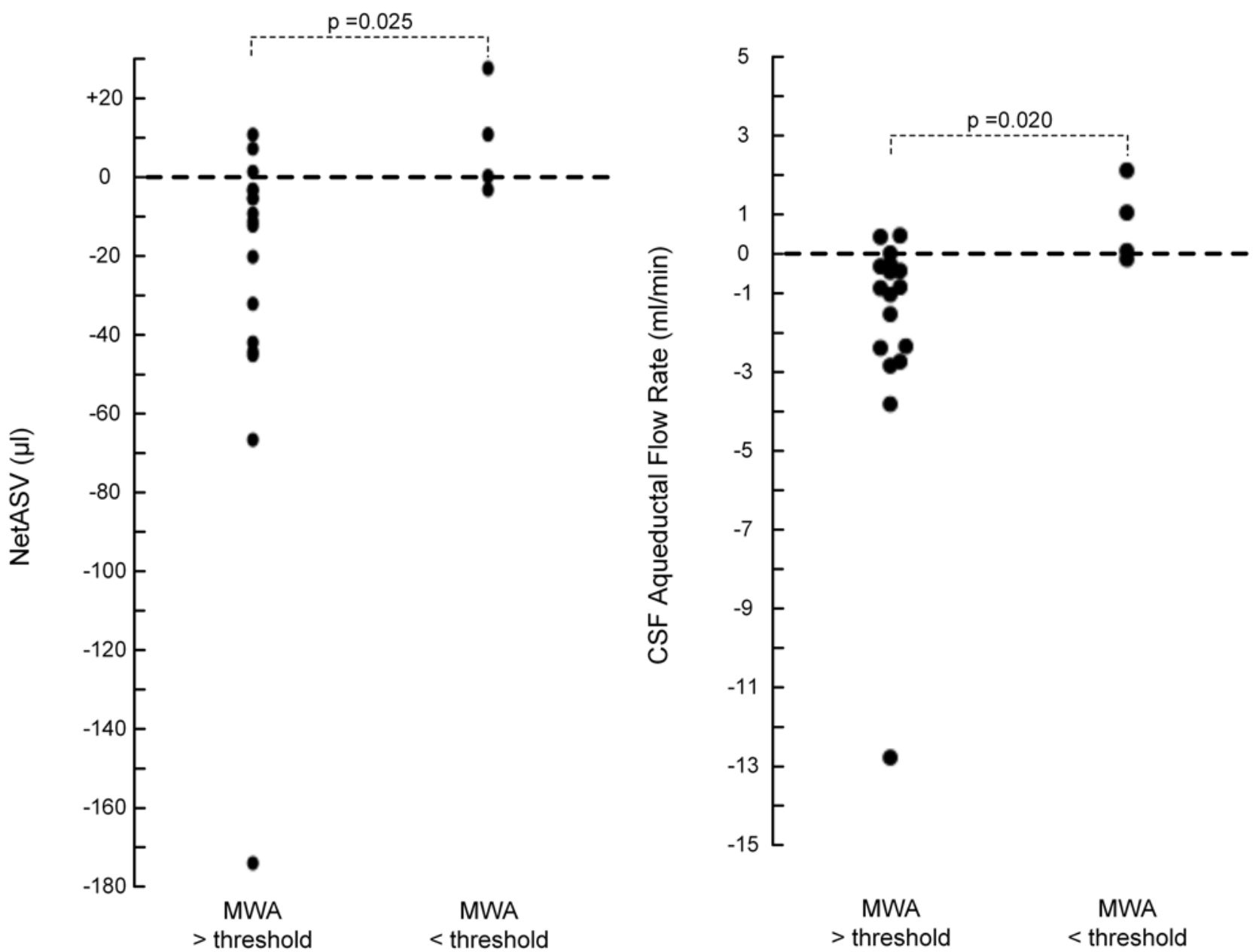

FIG. 3. Graphic presentation showing patients with iNPH with preoperative MWA values either above $(n=17)$ or below $(n=4)$ the thresholds used for selection of patients for shunting. Left: Net ASV values $(\mu \mathrm{l})$. Right: Estimated CSF aqueductal flow rates (ml/ $\mathrm{min})$. The $\mathrm{p}$ values for differences between MWA groups were determined by the Mann-Whitney test.

and retrograde directions would be superimposed on each other. ${ }^{25}$ However, good reproducibility has also been demonstrated for the PC-MRI technique for quantification of weak liquid oscillations such as at the aqueduct level. ${ }^{23,37}$ A phantom study showed an error of net flow estimates to be approximately $\pm 10 \%$ at typical aqueductal flow rates. ${ }^{39}$

Previous investigators have also demonstrated that the calculation of aqueductal stroke volume is less sensitive to inaccuracies from manual selection of ROIs than is the calculation of average flow velocity. 3,14

For estimations of CSF formation rate based on aqueductal flow rate, under the assumption that CSF is entirely produced in the choroid plexus of the supratentorial ventricles, variations have been shown at $\pm 23.1 \%$ on average with PC-MRI using a pixel size of $0.39 \mathrm{~mm} \times 0.39$ $\mathrm{mm},{ }^{25}$ and these variations were caused solely by manual definition of the aqueduct, which was compared and found inferior to automatic definition that also was immune to image noise. The pixel size in our study was moderate $(0.60 \mathrm{~mm} \times 0.80 \mathrm{~mm})$, and high-resolution flow measurements are indeed critical for measurement of net CSF flow over the cardiac cycle used for estimation of supratento- rial CSF formation. However, the large aqueductal lumen area associated with hydrocephalus causes more voxels to be included in the ROI and should therefore enhance measurement accuracy. In comparison with these previous studies, our PC-MRI technique also benefits from a higher magnetic field strength ( $3.0 \mathrm{~T}$ vs $1.5 \mathrm{~T})$, a similar or higher temporal resolution (30-40 vs 30 phases), and a thinner image slice (4 vs $6 \mathrm{~mm}$ ).

Previous studies of test-retest variability in aqueductal flow measurements have focused on healthy subjects. Such validations are also warranted for patients with hydrocephalus, where aqueductal flow is typically much larger than in subjects who are normocephalic. This was beyond the scope of this clinical study. However, the net flow measurements in our 2 healthy subjects demonstrated moderate, net antegrade flow, as could be expected based on results from previous studies. The results from our patient cohort also show a quite consistent tendency toward a moderate, but significant, reduction in retrograde aqueductal flow after shunt placement (Fig. 2). These observations might therefore indicate that inaccuracies due to measurement error are limited. 


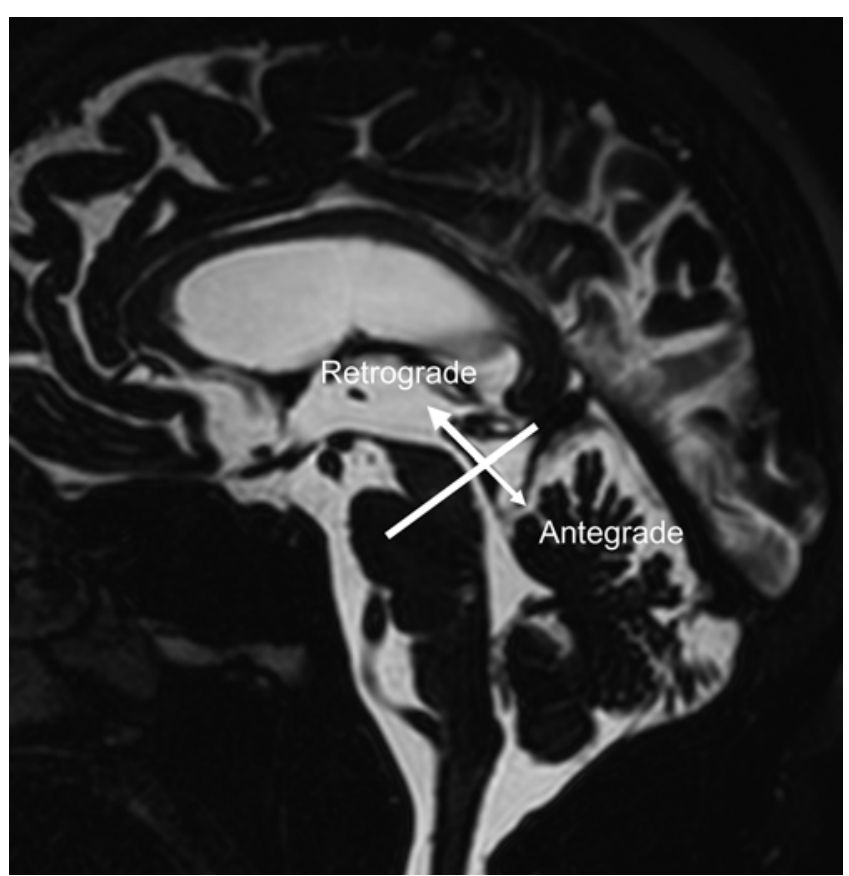

FIG. 4. Sagittal, heavily fluid-weighted MR image obtained with steadystate technique. Image illustrates that during 1 cardiac cycle CSF pulsates in both the antegrade (craniocaudal) and retrograde direction. Net retrograde flow was found in $82 \%$ of patients with iNPH and signs of reduced intracranial compliance from ICP monitoring before shunt placement.

\section{Other Study Limitations}

PC-MRI and invasive ICP monitoring for practical reasons could not be recorded simultaneously. PC-MRI was performed during the daytime, and ICP monitoring was performed overnight. It is well known that ICP, including MWA, fluctuates continuously over time due to physiological variations, and it has not been established in any study whether the PC-MRI data retrieved from a short time interval are representative of the daily average one would ideally want to measure.

\section{Conclusions}

In our study, retrograde net aqueductal flow was demonstrated to be a common phenomenon (82\%) in patients with iNPH and impaired intracranial compliance, and after shunt placement, net flow changed significantly in the antegrade direction. The observations may add new insight into the mechanisms behind ventricular enlargement in iNPH and how shunts work. In addition, the findings challenge conventional theories claiming that CSF production takes place mainly in the choroid plexus and resorption at the pacchionian granulations. This study also questions previous estimates of CSF production rates from invasive studies.

\section{Acknowledgments}

K. E. Emblem received a grant from the South-Eastern Norway Regional Health Authority.

\section{References}

1. Amiry-Moghaddam M, Frydenlund DS, Ottersen OP: An- choring of aquaporin- 4 in brain: molecular mechanisms and implications for the physiology and pathophysiology of water transport. Neuroscience 129:999-1010, 2004

2. Badaut J, Fukuda AM, Jullienne A, Petry KG: Aquaporin and brain diseases. Biochim Biophys Acta 1840:1554-1565, 2014

3. Bader C, Cyrille C, Jadwiga Z, Joel D, Fichten A, Catherine GJ, et al: Estimation of the lateral ventricles volumes from a 2D image and its relationship with cerebrospinal fluid flow. BioMed Res Int 215989:2013, 2013

4. Balédent O, Gondry-Jouet C, Meyer ME, De Marco G, Le Gars D, Henry-Feugeas MC, et al: Relationship between cerebrospinal fluid and blood dynamics in healthy volunteers and patients with communicating hydrocephalus. Invest Radiol 39:45-55, 2004

5. Bateman GA: Extending the hydrodynamic hypothesis in chronic hydrocephalus. Neurosurg Rev 28:333-334, 2005

6. Bateman GA, Brown KM: The measurement of CSF flow through the aqueduct in normal and hydrocephalic children: from where does it come, to where does it go? Childs Nerv Syst 28:55-63, 2012

7. Bateman GA, Napier BD: External hydrocephalus in infants: six cases with MR venogram and flow quantification correlation. Childs Nerv Syst 27:2087-2096, 2011

8. Bering EA Jr: Water exchange of central nervous system and cerebrospinal fluid. J Neurosurg 9:275-287, 1952

9. Bering EA Jr, Sato O: Hydrocephalus: Changes in formation and absorption of cerebrospinal fluid within the cerebral ventricles. J Neurosurg 20:1050-1063, 1963

10. Bloch O, Auguste KI, Manley GT, Verkman AS: Accelerated progression of kaolin-induced hydrocephalus in aquaporin4-deficient mice. J Cereb Blood Flow Metab 26:1527-1537, 2006

11. Bradley WG Jr, Scalzo D, Queralt J, Nitz WN, Atkinson DJ, Wong P: Normal-pressure hydrocephalus: evaluation with cerebrospinal fluid flow measurements at MR imaging. Radiology 198:523-529, 1996

12. Brinker T, Stopa E, Morrison J, Klinge P: A new look at cerebrospinal fluid circulation. Fluids Barriers CNS 11:10, 2014

13. Brodbelt A, Stoodley M: CSF pathways: a review. Br J Neurosurg 21:510-520, 2007

14. Chiang WW, Takoudis CG, Lee SH, Weis-McNulty A, Glick $\mathrm{R}$, Alperin N: Relationship between ventricular morphology and aqueductal cerebrospinal fluid flow in healthy and communicating hydrocephalus. Invest Radiol 44:192-199, 2009

15. Cserr HF, Harling-Berg CJ, Knopf PM: Drainage of brain extracellular fluid into blood and deep cervical lymph and its immunological significance. Brain Pathol 2:269-276, 1992

16. Cutler RW, Page L, Galicich J, Watters GV: Formation and absorption of cerebrospinal fluid in man. Brain 91:707-720, 1968

17. Dandy WE: Experimental hydrocephalus. Ann Surg 70:129142, 1919

18. Egeler-Peerdeman SM, Barkhof F, Walchenbach R, Valk J: Cine phase-contrast MR imaging in normal pressure hydrocephalus patients: relation to surgical outcome. Acta Neurochir Suppl 71:340-342, 1998

19. Eide PK: Demonstration of uneven distribution of intracranial pulsatility in hydrocephalus patients. J Neurosurg 109:912-917, 2008

20. Eide PK, Saehle T: Is ventriculomegaly in idiopathic normal pressure hydrocephalus associated with a transmantle gradient in pulsatile intracranial pressure? Acta Neurochir (Wien) 152:989-995, 2010

21. Eide PK, Sorteberg W: Association among intracranial compliance, intracranial pulse pressure amplitude and intracranial pressure in patients with intracranial bleeds. Neurol Res 29:798-802, 2007

22. Eide PK, Sorteberg W: Diagnostic intracranial pressure 
monitoring and surgical management in idiopathic normal pressure hydrocephalus: a 6-year review of 214 patients. Neurosurgery 66:80-91, 2010

23. Enzmann DR, Pelc NJ: Normal flow patterns of intracranial and spinal cerebrospinal fluid defined with phase-contrast cine MR imaging. Radiology 178:467-474, 1991

24. Graff-Radford NR, Godersky JC, Jones MP: Variables predicting surgical outcome in symptomatic hydrocephalus in the elderly. Neurology 39:1601-1604, 1989

25. Huang TY, Chung HW, Chen MY, Giiang LH, Chin SC, Lee CS, et al: Supratentorial cerebrospinal fluid production rate in healthy adults: quantification with two-dimensional cine phase-contrast MR imaging with high temporal and spatial resolution. Radiology 233:603-608, 2004

26. Iliff JJ, Wang M, Liao Y, Plogg BA, Peng W, Gundersen GA, et al: A paravascular pathway facilitates CSF flow through the brain parenchyma and the clearance of interstitial solutes, including amyloid $\beta$. Sci Transl Med 4:147ra111, 2012

27. Kim DS, Choi JU, Huh R, Yun PH, Kim DI: Quantitative assessment of cerebrospinal fluid hydrodynamics using a phase-contrast cine MR image in hydrocephalus. Childs Nerv Syst 15:461-467, 1999

28. Krauss JK, Droste DW, Vach W, Regel JP, Orszagh M, Borremans JJ, et al: Cerebrospinal fluid shunting in idiopathic normal-pressure hydrocephalus of the elderly: effect of periventricular and deep white matter lesions. Neurosurgery 39:292-300, 1996

29. Levine DN: Intracranial pressure and ventricular expansion in hydrocephalus: have we been asking the wrong question? J Neurol Sci 269:1-11, 2008

30. Linninger AA, Tsakiris C, Zhu DC, Xenos M, Roycewicz $P$, Danziger Z, et al: Pulsatile cerebrospinal fluid dynamics in the human brain. IEEE Trans Biomed Eng 52:557-565, 2005

31. Lorenzo AV, Page LK, Watters GV: Relationship between cerebrospinal fluid formation, absorption and pressure in human hydrocephalus. Brain 93:679-692, 1970

32. Nitz WR, Bradley WG Jr, Watanabe AS, Lee RR, Burgoyne B, O'Sullivan RM, et al: Flow dynamics of cerebrospinal fluid: assessment with phase-contrast velocity MR imaging performed with retrospective cardiac gating. Radiology 183:395-405, 1992

33. Penn RD, Basati S, Sweetman B, Guo X, Linninger A: Ventricle wall movements and cerebrospinal fluid flow in hydrocephalus. J Neurosurg 115:159-164, 2011

34. Ringstad G, Emblem KE, Geier O, Alperin N, Eide PK: Aqueductal stroke volume: comparisons with intracranial pressure scores in idiopathic normal pressure hydrocephalus. AJNR Am J Neuroradiol 36:1623-1630, 2015

35. Rubin RC, Henderson ES, Ommaya AK, Walker MD, Rall DP: The production of cerebrospinal fluid in man and its modification by acetazolamide. J Neurosurg 25:430-436, 1966

36. Sahar A, Hochwald GM, Ransohoff J: Alternate pathway for cerebrospinal fluid absorption in animals with experimental obstructive hydrocephalus. Exp Neurol 25:200-206, 1969

37. Wåhlin A, Ambarki K, Hauksson J, Birgander R, Malm J, Eklund A: Phase contrast MRI quantification of pulsatile volumes of brain arteries, veins, and cerebrospinal fluids compartments: repeatability and physiological interactions. J Magn Reson Imaging 35:1055-1062, 2012

38. Weller RO, Galea I, Carare RO, Minagar A: Pathophysiology of the lymphatic drainage of the central nervous system: Implications for pathogenesis and therapy of multiple sclerosis. Pathophysiology 17:295-306, 2010

39. Yoshida K, Takahashi H, Saijo M, Ueguchi T, Tanaka H, Fujita N, et al: Phase-contrast MR studies of CSF flow rate in the cerebral aqueduct and cervical subarachnoid space with correlation-based segmentation. Magn Reson Med Sci 8:91-100, 2009

\section{Disclosures}

Per Kristian Eide has a financial interest in the software company (dPCOM AS, Oslo) that manufactures the software (Sensometrics Software) used for analysis of the ICP recordings. Kyrre Eeg Emblem is a patent holder of NordicNeuroLab AS, Bergen, Norway.

\section{Author Contributions}

Conception and design: all authors. Acquisition of data: all authors. Analysis and interpretation of data: all authors. Drafting the article: all authors. Critically revising the article: all authors. Reviewed submitted version of manuscript: all authors. Approved the final version of the manuscript on behalf of all authors: Eide. Statistical analysis: all authors. Administrative/technical/material support: Ringstad. Study supervision: Eide, Emblem.

\section{Supplemental Information}

\section{Previous Presentations}

Portions of this work were presented in abstract form with oral presentation at the joint 64th meeting of the Scandinavian Neurosurgical Society/38th meeting of the Nordic Society of Neuroradiology in Reykjavik, Iceland, June 5-8, 2013.

\section{Correspondence}

Per Kristian Eide, Department of Neurosurgery, Oslo University Hospital-Rikshospitalet, Postboks 4950, Nydalen 0424 Oslo, Norway.email: peide@ous-hf.no. 